\title{
Ovarian Torsion During Pregnancy: A Case of
}

\section{Malignant Ovarian Cancer}

\author{
Latif Hacıoğlu*, Erbil Karaman, Deniz Dirik, Onur Karaaslan, Ali Kolusarı, Hanım Güler Şahin, \\ Abdulaziz Gül
}

Department of Gynecology and Obstetrics, Van Yuzuncu Yal University Dursun Odabas Medical Center, Van, Turkey

\begin{abstract}
Ovarian torsion is an acute and emergency condition during pregnancy. It is caused mainly by the benign cystic mass in the ovary. However, ovarian cancer may be seen as a cause which is very rare during pregnancy. Here, we aim to present a case of pregnant women with ovarian torsion and diagnosed with malignant ovarian tumor.
\end{abstract}

Key Words: Ovarian torsion, malignant ovarian cancer, pregnancy

\section{Case Report}

A 40 years old pregnant woman with 4 normal births and 2 abortions applied to our hospital with a sudden onset of abdominal pain and she was in her 25th week of pregnancy. In the USG examination, a $20 \mathrm{~cm} \times 18 \mathrm{~cm}$ multilobulated thick septal papillary cyst in the right adnexal area was observed. The left ovary was natural. $10 \mathrm{~cm}$ free liquid was observed in the abdomen. Tumor markers of the patient were as follows: CA-125: 194.5U/ml, CA-19-9: 1143,61U/ml, CEA: $0.71 \mathrm{ng} / \mathrm{ml}$ and hg: $11.1 \mathrm{~g} / \mathrm{dl}$, wbc 7.3 (103/ micl). In the MRI of the patient, a cyst with $101 \times 148 \times 119 \mathrm{~mm}$ multiloculated septa was observed. Since the patient had acute abdominal pain, an operation decision was taken. In the operation, approximately 2 liters of sero-mucinous fluid was observed in the abdomen, and a $20 \mathrm{~cm}$ cystic lesion was observed in the right ovary. Right oophorectomy was performed. The patient was diagnosed with grade 2 moderately differentiated mucinous carcinoma as a result of pathology. She did not accept the postoperative chemotherapy until the delivery. Delivery was made by cesarean at week 37. 7-8 Apgar score and $3400 \mathrm{~g}$ live baby boy were delivered, followed by a hysterectomy, unilateral oophorectomy and pelvic-paraaortic lymph node dissection, and apendectomy with omentectomy. The patient had no complications after the operation, and was discharged on the third postoperative day.
Conclusion: Although the incidence of ovarian cancer development during pregnancy is low, it should be kept in mind. Ovarian cancer may appear as ovarian torsion during pregnancy. Pregnancies complicated by ovarian cancer should be considered as a high-risk pregnancy and should be approached multidisciplinary.

\section{Introduction}

Ovarian torsion is an emergency condition that is caused by the rotation of ovarian vascular pedicles. It is usually occurred due to the ovarian masses either cystic or solid. The ovarian torsion should be kept in mind in case of acute abdominal pain during pregnancy especially after the first trimester of gestation. Its incidence is reported to be 1 in 5000 pregnancy (1). The main cause of ovarian torsion during the second trimester of pregnancy is the presence of ovarian mass either benign or malignant. The majority of cases are benign physiologic cysts or tumors including teratomas.

Most ovarian masses seen during pregnancy are functional and these masses are diagnosed early in routine obstetric USG controls. Although the vast majority of these masses are benign, and the possibility of cancer should not be ignored. The rate of adnexal masses during pregnancy is $1-6 \%$, making ovarian cancer the fifth most common cancer in pregnancy after breast, thyroid, cervical

${ }^{*}$ Corresponding Author: Latif Hacıoğlu, Assistant, Depatment of Obstetric and Gynecology, Van Yuzuncu Yil University Faculty of Medicine, 65200 Van, Turkiye

E-mail: dr.latifhacioglu123@hotmail.com, Telephone: 0 (507) 4725353

ORCID ID: Latif Hacioğlu: 0000-0001-5843-3237, Erbil Karaman: 0000-0003-1058-2748, Deniz Dirik: 0000-0002-5169-4052, Onur Karaaslan: 0000-0002-4599-1173, Ali Kolusar1: 0000-0001-5678-8958, Hanım Güler Şahin: 0000-0002-8596-0734, Abdulaziz Gül: 00000002-7097-3719 
cancer, and Hodgkin lymphoma (2-4). A retrospective review reported that ovarian cancer is the sixth most common cancer in the Asian population (5). Definitive diagnosis can only be made as a result of the pathological examination of a resected ovarian tissue of a qualified pathologist. However, the characteristic sonographic features of some benign ovarian masses are helpful in conjunction with MRI in USG.

Here, in this case report, we aimed to present a case of ovarian torsion which is diagnosed as musinous ovarian cancer. We also aimed to present the management strategy of our case from aspect of multidiciplinary approach.

\section{Case Report}

A 40-year-old pregnant woman with 4 normal births and 2 abortions applied to our hospital with a sudden onset of abdominal pain and she is in her 25 th week of pregnancy. She stated that the pain complaint has been occasional for a long time and increased in the last week. In the USG examination, a cystic structure with a $20 \mathrm{~cm}$ x 18 $\mathrm{cm}$ multilobulated thick septal and papillary extension was observed in the pregnancy and right adnexal area compatible with 25 weeks. The left ovary was observed naturally. $10 \mathrm{~cm}$ free liquid was observed in the deepest place in the abdomen. On laboratory examination, CA-125: $194.5 \mathrm{U} / \mathrm{ml}$, CA-19-9: $1143,61 \mathrm{U} / \mathrm{ml}$, CEA: $0.71 \mathrm{ng} / \mathrm{ml}$ and hg: $11.1 \mathrm{~g} / \mathrm{dl}$, wbc $7.3(103 / \mathrm{micl})$ were measured. In the MR image of the patient, a cystic lesion with multiloculated septa, which is hypohyperintense areas, was observed in the size of $101 \times 148 \times 119$ $\mathrm{mm}$. (Figure 1). After detailed information was given to the patient, the operation decision was taken and it was operated at the 25 th week of pregnancy. In the operation, approximately 2 liters of sero mucinous fluid was observed in the abdomen and aspirated. A cystic lesion of about $20 \mathrm{~cm}$ was observed in the right ovary. It was observed that the right ovary was torsioned. Right oophorectomy was performed. The cyst was sent to pathology and the patient was followed up. The patient was diagnosed with grade 2 moderately differentiated mucinous carcinoma, and chemotherapy was recommended at the multidisciplinary oncology council. However, the patient did not accept chemotherapy and was followed up. Delivery was made by cesarean at

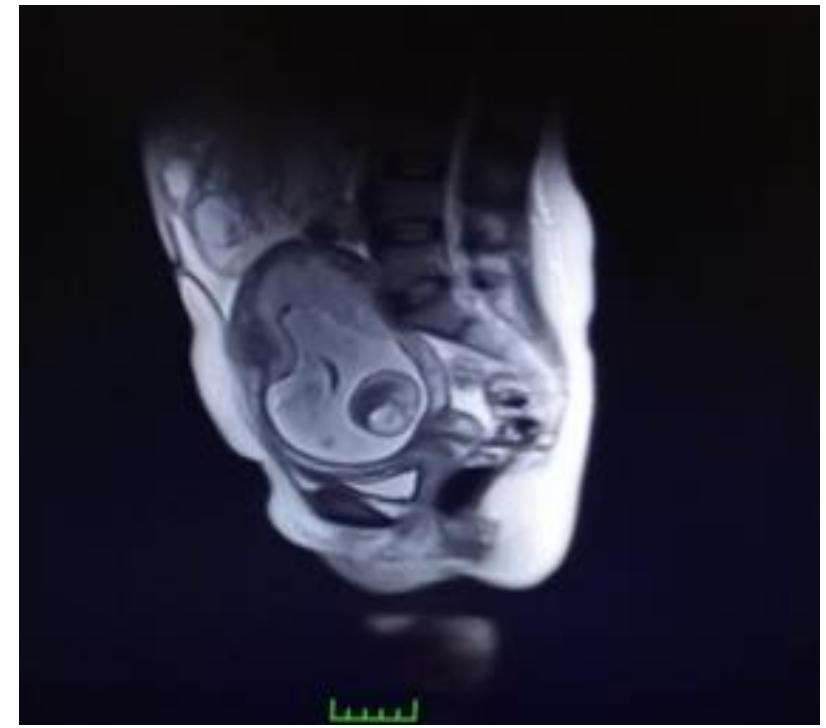

Fig. 1. MR image of the patient's cystic lesion

week 37. 7-8 Apgar score at 5th min. and $3400 \mathrm{gr}$ live baby boy was delivered, followed by hysterectomy unilateral oophorectomy and pelvicparaaortic lymph node dissection and appendectomy. There was no evidence of malignancy in the pathology report of the patient after definitive surgery. The patient had no complications after the operation and was discharged on the $3 \mathrm{rd}$ day after the operation. The patient was called for follow-up after 3-month intervals. It was observed that there was no recurrence in the third postoperative year.

\section{Discussion}

Diagnosis of malignant ovarian cancer during pregnancy is rare, requires accurate diagnosis and adequate treatment to achieve a good obstetric and oncological outcome. USG, MRI, and tumor markers are helpful in diagnosis (6). Epithelial ovarian tumors contain about half of all ovarian malignancies in pregnant women (7). There is no evidence that pregnancy worsens the prognosis of ovarian tumors compared to patients without pregnancy in terms of tumor histology, stage, and grade (8). Ovarian cancer can be managed by; pregnancy, stage of disease, future fertility desires, and the mother's desire to continue the pregnancy (9). The decision of the patient with a diagnosis of ovarian cancer in the first trimester to continue or terminate a pregnancy should be personalized and made by the fully informed patient in cooperation with the doctor.

The preoperative diagnosis of ovarian torsion is sometimes difficult in case of enlarged uterus. However, in the case of a huge ovarian mass, the diagnosis of torsion with malignant ovarian 
tumors is challenging. Surgery is indispensable in the treatment of malign ovarian tumors during pregnancy. Surgery during pregnancy is safe and is usually performed in the second trimester of gestation when the risk of abortion is low and there is still space to operative (10). Previous reports have shown that the incidence of negative fetal conditions (abortion, stillbirth, and preterm delivery) is significantly lower in laparoscopic surgery than in laparotomy, and the duration of surgery and hospitalization is significantly shorter (11). On the other hand, one of the most common complications of cancer in pregnant women is preterm birth, and the rate is up to $50 \%$. However, in our case, we did not see this complication. Our patients delivered at 37 th weeks of gestation.

Early termination of pregnancy does not improve the outcome of ovarian cancer (12). In pregnancy, the main principle to be considered when making the most appropriate treatment decision is the mother and fetal safety (13). The appropriate time for hysterectomy and secondary cytoreductive surgery is postpartum in order to eliminate the permanent disease in patients with advanced ovarian cancer diagnosed before delivery during pregnancy. This surgery can be performed with a cesarean section $(14,15)$. In our case, after cesarean delivery, hysterectomy, oophorectomy, and pelvic-paraaortic lymph node dissection were performed.

The vast majority of adnexal masses in pregnant women are benign and asymptomatic. It is detected incidentally in obstetric ultrasound examination. Although the incidence of ovarian cancer development during pregnancy is low, it should be kept in mind. Pregnancies complicated by ovarian cancer should be considered as a highrisk pregnancy and should be approached multidisciplinary. The best time for the operation is the second trimester. Simultaneous pregnancy does not affect the prognosis of cancer. The treatment decision should be made jointly by the informed patient and clinician, taking into account the mother and fetal condition.

\section{References}

1. Mancuso A, Broccio G, Angio LG, Pirri V. Adnexal torsion in pregnancy. Acta obstetricia et gynecologica Scandinavica 1997; 76: 83-84.

2. Hoover K, Jenkins TR. Evaluation and management of adnexal mass in pregnancy. American journal of obstetrics and gynecology 2011; 205: 97-102.
3. Leiserowitz GS. Managing ovarian masses during pregnancy. Obstetrical \& gynecological survey 2006; 61: 463-470.

4. Telischak NA, Yeh BM, Joe BN, Westphalen AC, Poder L, Coakley FV. MRI of adnexal masses in pregnancy. American Journal of Roentgenology 2008; 191: 364-370.

5. Shim MH, Mok C-W, Chang KH-J, et al. Clinical characteristics and outcome of cancer diagnosed during pregnancy. Obstetrics \& gynecology science 2016; 59: 1-8.

6. Fruscio R, de Haan J, Van Calsteren K, Verheecke M, Mhallem M, Amant F. Ovarian cancer in pregnancy. Best Practice \& Research Clinical Obstetrics \& Gynaecology 2017; 41: 108-117.

7. Runowicz CD, Brewer M. Adnexal mass in pregnancy. UpToDate (Accessed on February 26th, 2015). 2015.

8. Grimm D, Woelber L, Trillsch F, Amsberg GK-v, Mahner S. Clinical management of epithelial ovarian cancer during pregnancy. European Journal of Cancer 2014; 50: $963-$ 971.

9. Palmer J, Vatish M, Tidy J. Epithelial ovarian cancer in pregnancy: a review of the literature. BJOG: An International Journal of Obstetrics \& Gynaecology 2009; 116: 480-491.

10. Mazze RI, Kallén B. Reproductive outcome after anesthesia and operation during pregnancy: a registry study of 5405 cases. American journal of obstetrics and gynecology 1989; 161: 1178-1185.

11. Shigemi D, Aso S, Matsui H, Fushimi K, Yasunaga H. Safety of laparoscopic surgery for benign diseases during pregnancy: a Nationwide retrospective cohort study. Journal of minimally invasive gynecology 2019; 26: 501-506.

12. Stensheim H, Møller B, Van Dijk T, Fossa SD. Cause-specific survival for women diagnosed with cancer during pregnancy or lactation: a registry-based cohort study. Journal of Clinical Oncology 2009; 27: 45-51.

13. Pearl J, Price R, Richardson W, Fanelli R. Guidelines for diagnosis, treatment, and use of laparoscopy for surgical problems during pregnancy. Surgical endoscopy 2011; 25: 3479.

14. Méndez LE, Mueller A, Salom E, GonzálezQuintero VH. Paclitaxel and carboplatin chemotherapy administered during pregnancy for advanced epithelial ovarian cancer. Obstetrics \& Gynecology 2003; 102: 12001202.

15. Picone O, Lhommé C, Tournaire $M$, et al. Preservation of pregnancy in a patient with a stage IIIB ovarian epithelial carcinoma diagnosed at 22 weeks of gestation and treated with initial chemotherapy: case report and literature review. Gynecologic oncology 2004; 94: 600-604. 